\title{
Adherencia a la dieta mediterránea en escolares de Educación Primaria partícipes en actividad física: una revisión sistemática
}

\author{
Adherence to the Mediterranean Diet in Primary Education pupils involved \\ in physical activity: a systematic review \\ José Francisco López-Gil ${ }^{1}$, Edina Maria de Camargo² ${ }^{2}$ Juan Luis Yuste Lucas ${ }^{3}$ \\ 1 Facultad de Ciencias del Deporte, Universidad de Murcia (UM), San Javier, Región de Murcia, España. \\ 2 Programa de Pós-Graduação em Educação Física, Universidade Federal do Paraná (UFPR), Curitiba, Paraná, Brasil. \\ 3 Facultad de Educación, Universidad de Murcia (UM), Espinardo, Región de Murcia, España.
}

\author{
CORRESPONDENCIA: \\ José Francisco López-Gil \\ jf.lopezgil@um.es
}

Recepción: abril 2019 • Aceptación: febrero 2020

\section{CÓMO CITAR EL ARTÍCULO:}

López-Gil, J. F., Camargo, E. M., \& Yuste, J. L. (2020). Adherencia a la dieta mediterránea en escolares de Educación Primaria partícipes en actividad física: una revisión sistemática. Cultura, Ciencia y Deporte, 15(44), 267-275.

\section{Resumen}

En la actualidad, existe una clara tendencia del abandono del estilo de vida mediterráneo, en especial en niños y adolescentes. La dieta mediterránea se postula como una de las formas de alimentación más saludables que existen. Conocer la adherencia a la dieta mediterránea en escolares resulta de interés, ya que podrían desplegarse acciones para promover una alimentación saludable, especialmente desde la asignatura de Educación Física. Esta es una revisión sistemática de estudios transversales que determinaron la adherencia a la dieta mediterránea en escolares, mediante la búsqueda en cinco bases de datos distintas, entre 2014 y 2019. Para la selección de los artículos, fue preciso considerar el tipo de estudio, la franja etaria analizada, así como el lugar de realización de este. 13 estudios fueron incluidos en la revisión, donde el $69.2 \%$ de los mismos presenta que más de la mitad de la muestra de niños incluidos en los estudios necesitan mejorar sus patrones de alimentación (50\%). En los últimos años parecen haberse producido ligeras mejoras con relación a la adherencia al patrón mediterráneo por parte de los escolares. No obstante, se requiere un mayor número de estrategias de intervención para la promoción de hábitos alimenticios correctos.

Palabras clave: alimentación saludable; Patrón mediterráneo; Escolares; Niños.

\begin{abstract}
Today, there is a clear trend of abandonment of the Mediterranean lifestyle, especially in children and adolescents. Mediterranean diet is postulated as one of the healthiest forms of feeding that exist. Knowing the adherence to the Mediterranean diet in schoolchildren results interesting, since actions could be taken to promote a healthy diet, especially from the subject of Physical Education. Systematic review of cross-sectional studies that determined adherence to the Mediterranean diet in schoolchildren, by searching five different databases between 2014 and 2019. For the selection of the articles, it was necessary to consider the type of study, the age range analysed, as well as the location where the study was carried out. 13 studies were included in the review, where $69.2 \%$ of them show that more than half of the sample of children included in the studies need to improve their eating patterns $(50 \%)$. In recent years there seem to have been slight improvements in relation to adherence to the Mediterranean pattern by schoolchildren. However, a greater number of intervention strategies are required to promote correct eating habits.
\end{abstract}

Key words: Healthy eating; Mediterranean pattern; Schoolers; Children. 


\section{Introducción}

La prevalencia de sobrepeso y obesidad en población infantil y adolescente ha experimentado un aumento paulatino en las últimas tres décadas (Abarca-Gómez et al., 2017; Ng et al., 2014). Aunque se crea que este hecho pueda estar ligado únicamente a problemas genéticos o endocrinos, diferentes autores han determinado que estos casos suponen únicamente en torno al $2 \%$ de los mismos, siendo otros factores de mayor relevancia para el desarrollo de esta el ambiente obesogénico (sedentarismo, fast food, TV), factores familiares (padres obesos, bajo nivel socioeconómico), psicológicos (estrés, depresión) o alteraciones del sueño (insomnio) (Kumar \& Kelly, 2017).

Así, nos encontramos en la actualidad con diversas modificaciones que favorecen este ambiente obesogénico, como son: la forma en que se producen los alimentos y la creciente disponibilidad de estos (Popkin, 2006a), la elevada prevalencia de hábitos alimentarios no saludables (Popkin, 2006b), así como la disminución de la prevalencia de la actividad física (Hallal et al., 2012; Owen, Sparling, Healy, Dunstan, \& Matthews, 2010).

En referencia a los hábitos alimenticios, recientemente se ha puesto de manifiesto la imperante necesidad de lograr una mejora de estos a escala mundial (Afshin et al., 2019), puesto que una inadecuada alimentación constituye un factor de riesgo que causa más muertes que otros factores, como el tabaquismo (Gakidou et al., 2017).

En la misma línea, el patrón de alimentación de la población se ha visto modificado considerablemente debido al reemplazo de alimentos saludables por otros ricos en grasas y azúcares, con un alto nivel de procesamiento y, por ende, con una elevada densidad energética (Juul, Martínez-Steele, Parekh, Monteiro, \& Chang, 2018; Martins, Levy, Claro, Moubarac, \& Monteiro, 2009; Popkin, 2006b).

Al hilo de lo anterior, la dieta mediterránea (DM) se postula como una de las formas de alimentación más saludables que existen (Márquez-Sandoval, Bulló, Vizmanos, Agustench, \& Salvadó, 2008; De la Montaña, Castro, Cobas, Rodríguez, \& Míguez, 2012), debido a sus características particulares, como son: un elevado consumo de alimentos de procedencia vegetal (verduras, frutas, frutos secos, legumbres, frutas, frutos secos, granos enteros, aceite de oliva), cierta cantidad de lácteos (yogur y queso), un consumo bajo o moderado de carnes y pescados, un consumo moderado de vino, así como un estilo de vida activo (Mariscal-Arcas et al., 2009; Serra-Majem et al., 2004; Sofi, Macchi, Abbate, Gensini, \& Casini, 2014; Trichopoulou et al., 1995).
A pesar de ello, la adherencia a la DM en la región mediterránea ha desmejorado con el paso de los años (Vardavas, Linardakis, Hatzis, Saris, \& Kafatos, 2010), en especial entre aquellos más jóvenes (Arriscado, Muros, Zabala, \& Dalmau, 2014), pudiendo este hecho deteriorar la salud de estos, así como aumentar el riesgo de padecer enfermedades cardiovasculares, síndrome metabólico u obesidad (Mozaffarian, Appel, \& Van Horn, 2011; Arriscado et al., 2014). Igualmente, García y col. (2015) pusieron de manifiesto la clara tendencia del abandono del estilo de vida mediterráneo a través de una revisión sistemática minuciosa de estudios que determinaron la adherencia al patrón mediterráneo.

En esta línea, conocer la adherencia a la dieta mediterránea en escolares resulta de gran relevancia, puesto que, tanto el ámbito escolar, como especialmente el área de Educación Física, se postulan como óptimos escenarios para desplegar acciones dirigidas a la promoción de la alimentación saludable. Asimismo, dichas acciones pueden producir cambios positivos en la prevalencia de sobrecarga ponderal (suma de personas que padecen sobrepeso u obesidad), mejorando de manera significativa tanto las nociones como el consumo de alimentos salubres, así como reduciendo la ingesta de alimentos insalubres (Aguilar et al., 2011; BacardíGastón, Pérez-Morales, \& Jiménez-Cruz, 2012; Briones-Villalba, Gómez-Miranda, Ortiz-Ortiz, \& Rentería, 2018; Ratner et al., 2013; Vio, Salinas, Motenegro, González, \& Lera, 2014).

Con el objetivo de determinar la adherencia a la DM en niños y adolescentes fue diseñado y validado por Serra-Majem y col. (2004) el test KIDMED (Mediterranean Diet Quality Index in children and adolescents). Este test oscila entre 0 y 12 puntos, y se basa en una prueba de 16 preguntas que puede ser auto-administrada o realizada mediante entrevista por profesionales de la salud. A las preguntas que incluyen aspectos negativos con relación a la DM se les asigna un valor de -1; por el contrario, en aquellas que hacen referencia a aspectos positivos se puntúan con +1 . A partir del sumatorio de todos los valores de la prueba, se establecen tres niveles distintos: 1) > 8 puntos, dieta mediterránea óptima; 2) entre 4-7 puntos, se necesitan mejoras para adherirse al patrón mediterráneo; y 3 ) $\leq 3$, muy baja calidad de la dieta (Serra-Majem et al., 2004).

Finalmente, el objetivo de este trabajo de investigación fue conocer los resultados de la adherencia a la DM en escolares de Educación Primaria, o en niños que presentaran la edad que abarca esta etapa educativa, es decir, desde los 6 hasta los 12 años; que realizan actividad física (Educación Física, actividad física extraescolar, etc.). 


\section{Base de datos empleada}

Medline

Scopus

Web Of Science

Scielo Citation Index

Google Scholar
Palabras clave (Keywords)

Mediterranean Diet AND Adherence AND Primary Education.

Mediterranean Diet AND Adherence AND Primary Education.

Mediterranean Diet AND Adherence AND Primary Education.

Mediterranean Diet AND Adherence AND Primary Education.

Dieta Mediterránea AND Adherencia AND Educación Primaria.

"Mediterranean Diet"; "Adherence"; "Primary Education"; "Index"; "Score"

"Dieta Mediterránea"; "Adherencia"; "Educación Primaria"; "Índice"; "Puntuación";

Figura 1. Estrategia de búsqueda.

\section{Método}

Se realizó una revisión sistemática en torno a investigaciones de tipo transversal, tanto a nivel internacional como nacional, que evaluaron la adherencia a la DM en escolares de Educación Primaria de edades comprendidas en el mismo rango de edad (6 a 12 años). Dicha revisión fue realizada siguiendo los principios establecidos de la declaración PRISMA (Moher, Liberati, Tetzlaff, \& Altman, 2009).

\section{Estrategia de búsqueda}

La búsqueda fue realizada dentro de las principales bases de datos del ámbito de la salud y de Educación Física: Medline, Scopus, Web of Science, SciELO Citation Index y Google Scholar. Estas bases de datos se incluyeron porque son bases de datos de ciencias de la salud, esperando encontrar en las mismas artículos relacionados con la temática abordada. Fueron incluidos artículos publicados desde enero de 2014 hasta enero de 2019. Esta fecha fue estipulada debido a la existencia de una revisión sistemática publicada en la literatura con anterioridad que abarcó desde enero de 2000 hasta enero de 2014 (García et al., 2015). Para llevar a cabo la búsqueda estratégica se hizo uso de los siguientes descriptores en ciencias de la salud (DeCS): dieta mediterránea, adherencia y Educación Primaria; tanto en idioma castellano como en inglés (Figura 1).

Primeramente, fueron analizados los títulos y resúmenes de todos los artículos localizados de manera independiente por dos de los tres autores de este trabajo de investigación a través de la estrategia de búsqueda empleada. Asimismo, los revisores evaluaron independientemente los artículos completos y se procedió a la selección de estos, de acuerdo a los criterios de inclusión previamente establecidos. Además, las discrepancias entre los revisores se resolvieron con la ayuda del tercer autor, de acuerdo con las reglas de PRISMA (Moher et al., 2009).

Por otro lado, las referencias citadas en los estudios seleccionados fueron empleadas para obtener publica- ciones adicionales y no obviar informaciones reveladoras, mediante el procedimiento empleado. Además, algunas informaciones relevantes fueron solicitadas directamente a los autores. Por último, se procedió a un análisis exhaustivo de los artículos para establecer su integración o exclusión en esta revisión.

\section{Criterios de inclusión}

En cuanto a los criterios de inclusión, se hizo uso de los siguientes: 1) estudios de tipo transversal que determinaran la adherencia a la DM a través del test KIDMED; 2) estudios realizados en niños y adolescentes, siempre que estuvieran disponibles las informaciones para las edades de entre 6 y 12 años; 3) estudios llevados a cabo en colegios de Educación Primaria; 4) estudios con escolares que realizan actividad física (Educación Física, actividad física extraescolar, etc.).

\section{Criterios de exclusión}

Con relación a los criterios de exclusión, fueron empleados los citados a continuación: 1) estudios centrados únicamente sobre escolares con alguna enfermedad o patología específica; 2) trabajos de investigación de tesis doctorales, así como trabajos finales de grado o máster y artículos de opinión.

\section{Gestión de la información}

Los artículos presentes en esta revisión fueron colocados y resumidos en autor y año de publicación, país, tamaño de la muestra, edad y resultados sobre la adherencia a la DM.

\section{Resultados}

En la Figura 2 se muestran los distintos procesos de la revisión sistemática efectuada, donde fueron encontrados un total de 708 artículos (100.00\%) durante la fase de identificación. En la fase de revisión fueron excluidos 


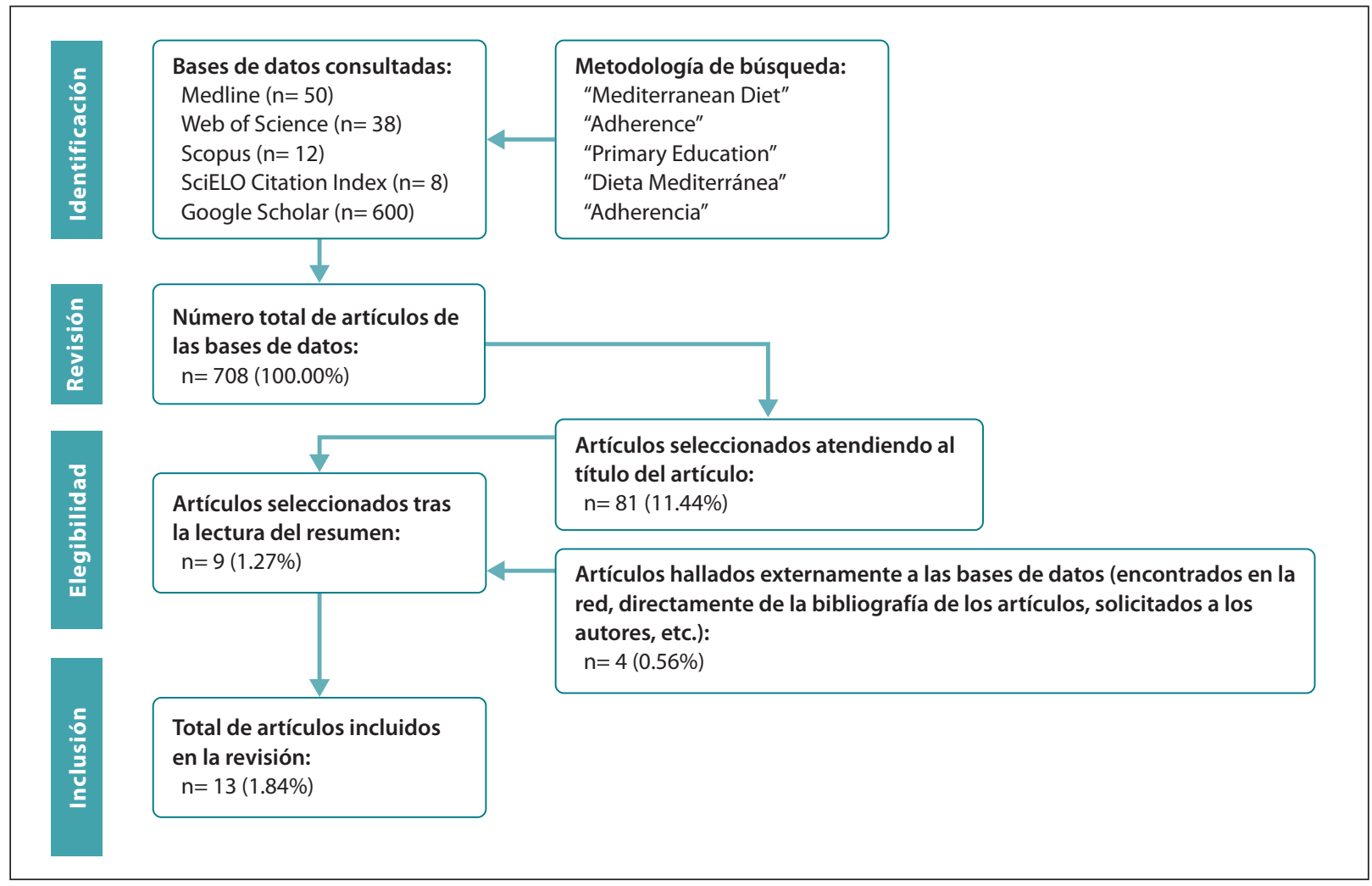

Figura 2. Diagrama de flujo sobre el proceso de selección de los artículos.

627 artículos (88.56\%) por presentar un título no relacionado con la temática de estudio. Posteriormente, en la fase de elegibilidad, fueron descartados 72 artículos (10.17\%), 50 de los mismos (7.06\%) por estar enfocados sobre escolares con alguna enfermedad/patología concreta, y 22 estudios (3.11\%) por ser trabajos de fin de grado, máster, etc. Finalmente, en la fase inclusión, fueron seleccionados un total de 13 artículos (1.84\%); 9 artículos (1.27\%) tras la lectura del resumen/abstract y 4 estudios $(0.56 \%)$ hallados de manera externa. Todo lo anterior, atendiendo siempre a los criterios de exclusión e inclusión previamente mencionados.

En la Tabla 1 se muestran las características de los estudios encontrados en la búsqueda realizada, incluyendo el autor/es, el año de publicación, el tamaño y edad de la muestra, así como los resultados de adherencia a la DM; tanto a nivel global, como estratificados por género y nivel de adherencia, siempre que fuese posible (Tabla 2).

En total, 13 estudios fueron incluidos en la revisión, siendo: siete de España, dos de Chile, uno de Colombia, uno de Grecia, y uno de Italia. El período de publicación fue de enero de 2014 a enero de 2019. Todos los estudios fueron transversales (criterio de inclusión) y las muestras variaron de 298 participantes hasta 1176. Todos investigaron ambos sexos (masculino y femenino) (Tabla 1).
Sobre la adherencia a la DM en niños, nueve estudios estratificaron los resultados por sexo, mientras que tres presentaron datos globales (Tabla 2). Además, la adherencia a la DM fue clasificada en: "necesita mejorar" considerando, de manera conjunta, las categorías de baja y moderada adherencia a la DM, y "no necesita mejorar", incluyendo una óptima adherencia a la DM, de acuerdo con el tamaño muestral y proporción de cada estudio incluido en la revisión (Figura 3). Así, considerando los 13 estudios incluidos en la revisión, el $69.2 \%$ de los mismos presenta que más de la mitad de la muestra de niños incluidos en los estudios necesitan mejorar sus patrones de alimentación (50\%).

\section{Discusión}

La síntesis de los estudios incluidos en esta revisión permite conocer informaciones relevantes sobre la adherencia de la DM en niños a través del test KIDMED, ya que, a través del seguimiento de este patrón alimentario, los escolares pueden ser capaces de disminuir o de mantener un peso corporal adecuado, contribuyendo a una mejor salud en estas edades $y$, de manera inexorable, una reducción de los alarmantes niveles de prevalencia de sobrepeso y obesidad con los que nos encontramos actualmente. 


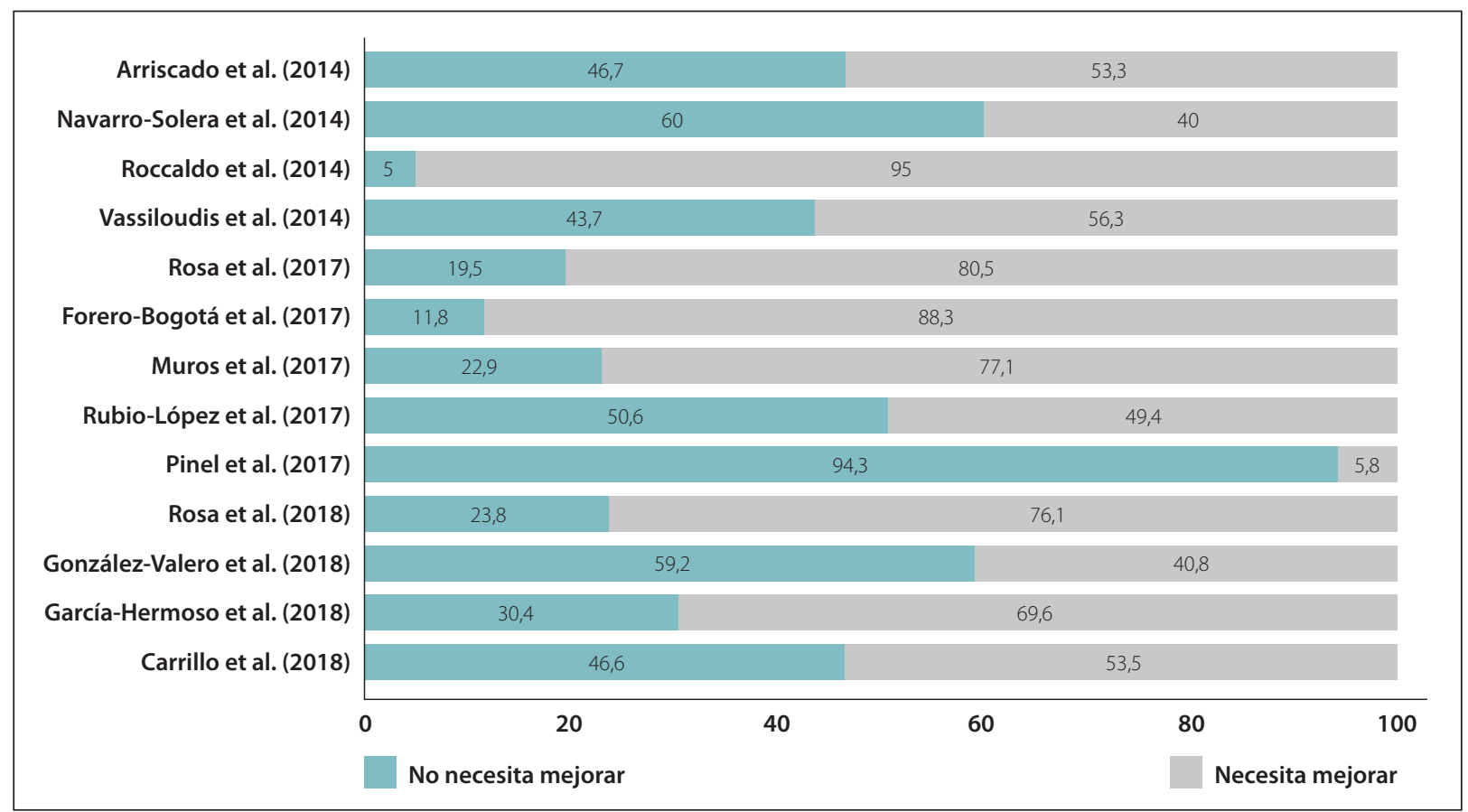

Figura 3. Adherencia a la DM en función del test KIDMED en escolares de 6 a 12 años $(n=13)$.

Tabla 1. Características de los estudios incluidos en la revisión $(n=13)$.

\begin{tabular}{|c|c|c|c|}
\hline Autor (Año) & Lugar & Muestra (Sexo) & Edad (Años) \\
\hline Arriscado et al. (2014) & Logroño (España) & $321(158+$ y $163 \precsim)$ & $11-12$ \\
\hline Navarro-Solera et al. (2014) & Valencia (España) & $\begin{array}{c}777(389 q \text { y } 388 \precsim) \\
683(\mathrm{~S} / \mathrm{E})\end{array}$ & $\begin{array}{l}8-16 * \\
(8-12)\end{array}$ \\
\hline Roccaldo et al. (2014) & Italia & $1740(840$ & $8-9$ \\
\hline Vassiloudis et al. (2014) & Atenas (Grecia) & $528(272+$ y $256 \precsim)$ & $10-12$ \\
\hline Forero-Bogotá et al. (2017) & Colombia & $\begin{array}{l}1118(610+\text { y } 508 \precsim) \\
442(246+\text { y } 196 \curvearrowright)\end{array}$ & $\begin{array}{l}9-18^{*} \\
(9-12)\end{array}$ \\
\hline Muros et al. (2017) & Santiago (Chile) & $515(255 q$ y $260 \precsim)$ & $10-11$ \\
\hline Rubio-López et al. (2017) & Valencia (España) & $1176(615+$ y $561 \gtrsim)$ & $6-9$ \\
\hline Pinel et al. (2017) & Granada (España) & $315\left(177+\right.$ y $\left.138{ }^{\lambda}\right)$ & $10-12$ \\
\hline Rosa et al. (2017) & Murcia (España) & $298(159+$ y $139 \precsim)$ & $8-12$ \\
\hline Carrillo et al. (2018) & Murcia (España) & $\begin{array}{l}634(354 q \text { y } 280 \precsim) \\
260(120 q \text { y } 140 \precsim)\end{array}$ & $\begin{array}{l}6-18 * \\
(6-12)\end{array}$ \\
\hline García-Hermoso et al. (2018) & Santiago (Chile) & 1140 (346 + y $\left.794{ }^{\circledR}\right)$ & $8-12$ \\
\hline González-Valero et al. (2018) & Granada (España) & $577(249+$ y $328 \gtrsim)$ & $10-12$ \\
\hline Rosa et al. (2018) & Murcia (España) & $\begin{array}{l}520(301+\text { y } 219 ð) \\
257(301+\text { y } 219 ð)\end{array}$ & $\begin{array}{l}8-17 * \\
(8-12)\end{array}$ \\
\hline
\end{tabular}

*Fueron seleccionadas únicamente las informaciones referidas a la franja de edad de 6-12 años.

Cuando se categoriza en "necesita mejorar" y "no necesita mejorar" la adherencia a la dieta mediterránea, el $69.2 \%$ de los estudios indican que más del $50 \%$ de los escolares incluidos en los estudios necesitan mejorar sus hábitos alimentarios.

En cuanto a la revisión de García y col. (2015), que investigó estudios publicados en el período compren- dido entre enero de 2000 hasta enero de 2014, la tasa global reveló que el 21\% de la población analizada presentaba una baja adherencia a la DM frente al $10 \%$ con alta adherencia, por lo que parece haber mejorado en los últimos cinco años.

En esta misma línea, Arriscado y col. (2014) realizaron un estudio para describir la adherencia a la DM en 
Tabla 2. Resultados para la adherencia a la DM en los estudios incluidos en la revisión $(n=13)$.

\begin{tabular}{|c|c|}
\hline Autor (Año) & Adherencia da DM \\
\hline $\begin{array}{c}\text { Arriscado et } \\
\text { al. (2014) }\end{array}$ & $\begin{array}{l}\text { Total = Baja } 4.7 \% \text {, Moderada } 48.6 \%, \text { Alta } 46.7 \% \\
+=\text { Baja } 3.1 \%, \text { Moderada } 49.7 \% \text {, Alta } 47.2 \% \\
\delta=\text { Baja } 6.1 \% \text {, Moderada } 46.4 \% \text {, Alta } 47.3 \%\end{array}$ \\
\hline $\begin{array}{l}\text { Navarro- } \\
\text { Solera et al. } \\
\quad(2014)\end{array}$ & $\begin{array}{l}\text { Total = Baja } 13 \% \text {, Moderada } 27 \% \text {, Alta } 60 \% \\
+=\text { Baja } 10.9 \% \text {, Moderada } 26.2 \% \text {, Alta } 63 \% \\
\hat{T}=\text { Baja } 14.8 \% \text {, Moderada } 27.7 \% \text {, Alta } 57.7 \%\end{array}$ \\
\hline $\begin{array}{l}\text { Roccaldo et } \\
\text { al. (2014) }\end{array}$ & $\begin{array}{l}\text { Total = Baja 32.8\%, Moderada } 62.2 \% \text {, Alta } 5.0 \% \\
+=\text { Baja } 32 \% \text {, Moderada } 62.8 \% \text {, Alta } 5.2 \% \\
\partial=\text { Baja } 33.6 \% \text {, Moderada } 61.5 \% \text {, Alta } 4.9 \%\end{array}$ \\
\hline $\begin{array}{l}\text { Vassiloudis } \\
\text { et al. (2014) }\end{array}$ & $\begin{array}{l}\text { Total = Baja } 4.4 \% \text {, Moderada 51.9\%, Alta } 43.7 \% \\
+=\text { Baja } 4.4 \% \text {, Moderada } 52.9 \% \text {, Alta } 42.6 \% \\
{ }^{\lambda}=\text { Baja } 4.3 \% \text {, Moderada } 50.8 \% \text {, Alta } 44.9 \%\end{array}$ \\
\hline $\begin{array}{l}\text { Forero- } \\
\text { Bogotá et } \\
\text { al. (2017) }\end{array}$ & $\begin{array}{l}\text { Total = Baja } 28.7 \% \text {, Moderada } 59.6 \% \text {, Alta } 11.8 \% \\
+=\text { Baja } 29.7 \% \text {, Moderada } 58.5 \% \text {, Alta } 11.8 \% \\
\delta=\text { Baja } 27.6 \% \text {, Moderada } 60.7 \% \text {, Alta } 11.7 \%\end{array}$ \\
\hline $\begin{array}{c}\text { Muros et al. } \\
(2017)\end{array}$ & Total = Baja 9.5\%, Moderada $67.6 \%$, Alta $22.9 \%$ \\
\hline $\begin{array}{l}\text { Rubio- } \\
\text { López et al. } \\
\text { (2017) }\end{array}$ & $\begin{array}{l}\text { Total = Baja 5.4\%, Moderada } 44 \% \text {, Alta } 50.6 \% \\
+=\text { Baja } 4.7 \% \text {, Moderada } 43.7 \% \text {, Alta } 51.5 \% \\
\delta=\text { Baja } 6.1 \% \text {, Moderada } 44.4 \% \text {, Alta } 49.6 \%\end{array}$ \\
\hline $\begin{array}{l}\text { Pinel et al. } \\
\text { (2017) }\end{array}$ & $\begin{array}{l}\text { Total = Baja 0.3\%, Moderada 5.5\%, Alta } 94.3 \% \\
+=\text { Baja 0.6\%, Moderada } 7.3 \% \text {, Alta } 92.1 \% \\
=\text { Baja } 0 \% \text {, Moderada } 3.6 \% \text {, Alta } 96.4 \%\end{array}$ \\
\hline $\begin{array}{l}\text { Rosa et al. } \\
\text { (2017) }\end{array}$ & $\begin{array}{l}\text { Total = Baja 39.5\%, Moderada } 41 \% \text {. Alta } 19.5 \% \\
+=\text { Baja } 40.9 \% \text {, Moderada } 39.6 \% \text {, Alta } 19.5 \% \\
\delta=\text { Baja } 38.1 \% \text {, Moderada } 42.4 \% \text {, Alta } 19.4 \%\end{array}$ \\
\hline $\begin{array}{l}\text { Carrillo et } \\
\text { al. (2018) }\end{array}$ & Total = Baja 24\%, Moderada $29.5 \%$, Alta $46.6 \%$ \\
\hline $\begin{array}{l}\text { García- } \\
\text { Hermoso et } \\
\text { al. (2018) }\end{array}$ & $\begin{array}{l}\text { Total = Baja 10.9\%, Moderada 58.7\%, Alta } 30.4 \% \\
+=\text { Baja } 12.4 \% \text {, Moderada 58.6\%, Alta } 28.9 \% \\
\hat{T}=\text { Baja } 9.3 \% \text {, Moderada, 58.8\%, Alta, } 31.9 \%\end{array}$ \\
\hline $\begin{array}{l}\text { González- } \\
\text { Valero et al. } \\
\quad(2018)\end{array}$ & Total = Baja-Moderada 40.8\%, Alta 59.2\% \\
\hline $\begin{array}{l}\text { Rosa et al. } \\
\quad(2018)\end{array}$ & $\begin{array}{l}\text { Total = Baja } 23.8 \% \text {, Moderada } 33 \% \text {, Alta } 43.1 \% \\
q=\text { Baja } 22.7 \% \text {, Moderada } 28.4 \% \text {, Alta } 39.5 \% \\
\partial=\text { Baja } 24.9 \% \text {, Moderada } 35.7 \% \text {, Alta } 46.7 \%\end{array}$ \\
\hline
\end{tabular}

una ciudad del norte de España. Por ello, fue incluida una muestra representativa de 321 escolares (163 niños y 158 niñas) de entre 11 y 12 años, provenientes de 31 escuelas de Logroño. Haciendo referencia a los resultados, se encontró una alta adherencia para el $46.7 \%$ (en $47.2 \%$ en niños y en $45.8 \%$ niñas); una moderada adherencia del $48,6 \%$ (46.4\% en niños y $49.7 \%$ en niñas); y una baja adherencia del $4.7 \%$ (6.1\% para el género masculino y $3.1 \%$ para el femenino). Finalmente, los autores señalaron que casi la mitad de la población de estudio se adhiere a la DM. Cuatro años después, en el estudio de González-Valero y col. (2018) se pretendió determinar las características y correla- ciones entre los niveles de actividad física, la adherencia a la DM, el uso problemático de videojuegos y el autoconcepto de los alumnos de primaria. Así, participó una muestra de 577 escolares (328 niños y 249 niñas) de entre 10 y 12 años de Granada. Los resultados indicaron una óptima adherencia para el 59.2\% del total, y una necesidad de mejora para el 40,8\% de la muestra, concluyendo los autores que la mitad de ellos necesita mejorar el cumplimiento de la DM.

Por otro lado, Roccaldo y col. (2014) llevaron a cabo el estudio ZOOM8 en Italia, con el objetivo de determinar el grado de adherencia a la DM, comparando los resultados con los de otros países europeos. Los autores concluyeron que la adherencia óptima a la DM fue muy baja en escolares italianos. Igualmente, Vassiloudis y col. (2014) investigaron sobre los posibles vínculos entre la adherencia a la DM, el exceso de peso corporal, las conductas en cuanto al balance energético y el rendimiento académico. Los autores resaltaron la pobre adherencia a la DM por parte de los escolares griegos.

Por otra parte, Navarro-Solera, González-Carrascosa, y Soriano (2014) concluyeron que la adherencia a la DM tiene una asociación significativa con el estado nutricional de los jóvenes, empeorando en función del aumento de la edad. Rosa y col. (2017), en un estudio para analizar la relación entre la actividad física, la condición física y la calidad de la dieta, concluyeron que la actividad física, la condición física y la calidad de la dieta se relacionan de manera positiva. Pinel y col. (2017) desplegaron un estudio para analizar y relacionar el género con los parámetros de obesidad, actividades sedentarias y físicas y la calidad de la dieta. A raíz de estos resultados los autores resaltaron la necesidad de realizar intervenciones para motivar la realización de deporte, sobre todo en el sector femenino, además de concienciar a los jóvenes de las consecuencias del sedentarismo y la obesidad.

En línea de lo anterior, Rosa y col. (2018) acometieron un estudio para analizar la relación entre el estado de peso, la actividad física y la DM en escolares, seleccionando para ello 520 estudiantes (219 niños y 301 niñas) con una edad media de $12.81 \pm 2.99$, provenientes de tres centros educativos de carácter público de la Región de Murcia. Con relación a los resultados, y tomando la muestra de interés, se obtuvo una prevalencia baja para el $23.8 \%$ (24.9\% en niños y $22.7 \%$ en niñas), moderada para el 33\% (35.7\% para niños y $28.4 \%$ para niñas), y baja para el $43.1 \%$ (46.7\% en el género masculino y $39.5 \%$ en el femenino).

Sintetizar las informaciones sobre la adherencia a la DM parece importante para comprender mejor la alimentación de niños en edad escolar, siendo esta una 
etapa crucial para asentar hábitos que perduren a lo largo de la vida. Aunque existen diferentes procedimientos para evaluar el nivel de adhesión a la DM, el test KIDMED es una herramienta eficaz para evaluar la calidad de los hábitos alimentarios en los niños, así como en adolescentes. La prueba se basa en los principios del patrón de alimentación mediterráneo, siendo un instrumento fácil de cumplimentar por el entrevistado y fácil de evaluar por el entrevistador. Así, a través de la evaluación de 16 ítems, ayuda a identificar a personas con hábitos alimenticios poco saludables (baja puntuación), a sujetos con cierta adherencia pero que necesitan mejorar sus hábitos (puntuación media), y a individuos que presentan unos hábitos alimenticios adecuados (alta puntuación).

\section{Limitaciones y recomendaciones}

Aunque la validez y fiabilidad del test KIDMED está bien respaldada por la literatura (Mariscal-Arcas et al., 2009; Serra-Majem et al., 2004), a lo largo de la franja temporal empleada para localizar los artículos de esta revisión sistemática es posible observar la falta de estudios transversales en el ámbito escolar que han hecho uso del test KIDMED. Por ello, consideramos importante recomendar la realización de un mayor número de estudios, dirigidos principalmente a evaluar los hábitos alimenticios de los escolares como objetivo prioritario, ya que, esta franja etaria conforma una excelente oportunidad para el trabajo y promoción de hábitos alimenticios saludables que perduren a lo largo de la vida.

Además, pudimos comprobar la falta de homogeneidad en el uso de los instrumentos para evaluar dichas acciones, haciendo uso de otras herramientas como cuestionarios de frecuencia alimentaria, recordatorios de 24 horas o el Mediterranean Dietary Score (Trichopoulou et al., 1995), lo que se traduce en una dificultad para tener conciencia de la realidad con la que nos encontramos, así como la poca viabilidad de que los resultados de los estudios sean comparados.

En otro orden de ideas, otra limitación encontrada fue el hecho de que algunos estudios no mostraran los resultados estratificados por las distintas categorías que el test presenta, es decir, agrupando dos categorías en una sola (González-Valero et al., 2018); asimismo, también se encontró en otros estudios esta misma limitación, pero aplicada a la estratificación por género (Carrillo, García \& Rosa, 2018; González-Valero et al., 2018; Muros, Cofre-Bolados, Arriscado, Zurita \& Knox, 2017).

Por otro lado, es posible que existan artículos en la literatura sobre el tema analizado que no aparezcan incluidos en esta revisión sistemática. Este hecho se debe a que se siguieron los procedimientos de la declaración PRISMA (Moher et al., 2009), que establece que solo deben incluirse los artículos encontrados a través de los descriptores seleccionados, en las bases de datos mencionadas, con los criterios de inclusión y exclusión establecidos, tal y como se indica en la metodología del estudio. Así, la adopción de este procedimiento reduce la selección y el sesgo de resultados.

Por otra parte, aunque el procedimiento PRISMA requiere que se indique la fuerza de evidencia de los estudios, debido a la falta de información en los mismos para calcularla, dicha información no se incluyó en la presente revisión. En esta línea, es importante que los futuros estudios incluyan fehacientemente informaciones sobre la magnitud de evidencia científica, con el objetivo de mantener el rigor metodológico.

Los autores recomiendan estrategias de intervención en esta franja etaria, tanto con los discentes como con las familias, puesto que los padres son responsables de la alimentación de los más jóvenes. Estrategias efectivas sobre alimentación saludable podrían influir positivamente en el devenir de la salud de los escolares al llegar a la edad adulta.

\section{Conclusiones}

Aunque se requiere un mayor número de estudios sobre la temática analizada, la revisión de la literatura científica durante los últimos cinco años muestra ligeras mejoras con relación a la adherencia al patrón mediterráneo por parte de los escolares en comparación con la franja temporal previamente analizada por otros autores. No obstante, las cifras relativas al sobrepeso y la obesidad continúan siendo muy alarmantes. En esta línea, la escuela y, en especial, las sesiones de Educación Física se postulan como un escenario óptimo para llevar a cabo intervenciones en aras de promocionar hábitos alimenticios beneficiosos entre los más jóvenes. 
Abarca-Gómez, L., Abdeen, Z. A., Hamid, Z . A., Abu-Rmeileh, N. M., Acosta-Cazares, B., Acuin, C., ..., \& Ezzati, M. (2017). Worldwide trends in body-mass index, underweight, overweight, and obesity from 1975 to 2016: a pooled analysis of 2416 population-based measurement studies in 128.9 million children, adolescents, and adults. The Lancet, 390(10113), 2627-42. doi:10.1016/S0140-6736(17)32129-3

Aguilar, M. J., González, E., García, C. J., García, P. A., Álvarez, J., Padilla C. A., ..., \&, Ocete, E. (2011). Obesidad de una población de escolares de Granada: evaluación de la eficacia de una intervención educativa. Nutrición Hospitalaria, 26(3), 636-41. doi:10.3305/nh.2011.26.3.5195

Arriscado, D., Dalmau, J. M., Muros, J. J., \& Zabala, M. (2014). Relación entre condición física y composición corporal en escolares de primaria del norte de España. Nutrición Hospitalaria, 30(2), 385-94. doi:10.3305/nh.2014.30.2.7217

Afshin, A., Sur, P. J., Fay, K. A., Cornaby, L., Ferrara, G., Salama, J. S., ..., \& Murray, C. J. L. (2019). Health effects of dietary risks in 195 countries, 1990-2017: A systematic analysis for the Global Burden of Disease Study 2017. The Lancet, 393(10184), 1958-72. doi:10.1016/ S0140-6736(19)30041-8

Bacardí-Gascón, M., Pérez-Morales, M. E., \& Jiménez-Cruz, A. (2012). Intervención aleatorizada de seis meses en las escuelas, con un seguimiento de 18 meses para la prevención de obesidad infantil en escuelas primarias de México. Nutrición Hospitalaria, 27(3), 755-62. doi:10.3305/nh.2012.27.3.5756

Briones-Villalba, R. A., Gómez-Miranda, L. M., Ortiz-Ortiz, M., \& Rentería, I. (2018). Efecto de un programa de actividad física y educación nutricional para reducir el consumo de bebidas azucaradas y desarrollo de la obesidad en escolares de Tijuana, México. Revista Española de Nutrición Humana y Dietética, 22(3), 235. doi:10.14306/renhyd.22.3.519

Carrillo, P. J., García, E., \& Rosa, A. (2018). Estado nutricional y adherencia a la dieta mediterránea en escolares de la Región de Murcia. Perspectivas en Nutrición Humana, 20(2), 157-69. doi:10.17533/udea. penh.v20n2a04

De la Montaña, J., Castro, L., Cobas, N., Rodríguez, M., \& Míguez, M. (2012). Adherencia a la dieta mediterránea y su relación con el índice de masa corporal en universitarios de Galicia. Nutrición clínica y dietética hospitalaria, 32(3), 72-80. Recuperado de: http://www.nutricion. org/publicaciones/revista_2012_32_3/ADHERENCIA.pdf

Forero-Bogotá, M., Ojeda-Pardo, M., García-Hermoso, A., Correa-Bautista, J., González-Jiménez, E., Schmidt-RíoValle, J., ..., \& RamírezVélez, R. (2017). Body Composition, Nutritional Profile and Muscular Fitness Affect Bone Health in a Sample of Schoolchildren from Colombia: The Fuprecol Study. Nutrients, 9(2), 106. doi:10.3390/ nu9020106

Gakidou, E., Afshin, A., Abajobir, A. A., Abate, K. H., Abbafati, C., Abbas, K. M., ..., \& Murray, C. J. L. (2017). Global, regional, and national comparative risk assessment of 84 behavioural, environmental and occupational, and metabolic risks or clusters of risks, 1990-2016: A systematic analysis for the Global Burden of Disease Study 2016. The Lancet, 390(10100), 1345-422. doi:10.1016/S0140-6736(17)32366-8

García, S., Herrera, N., Rodríguez, C., Nissensohn, M., Román-Viñas, B., \& Serra-Majem, L. (2015). KIDMED test; prevalence of low adherence to the Mediterranean Diet in children and young; a systematic review. Nutrición Hospitalaria, 32(6), 2390-9. doi:10.3305/ nh.2015.32.6.9828

González-Valero, G., Ubago-Jiménez, J. L., Zurita-Ortega, F., ChacónCuberos, R., Castro-Sánchez, M., \& Puertas-Molero, P. (2018). Eating Habits and Lifestyles in Schoolchildren from Granada (Spain). A Pilot Study. Education Sciences, 8(4), 216. doi:10.3390/educsci8040216

Hallal, P. C., Andersen, L. B., Bull, F. C., Guthold, R., Haskell, W., \& Ekelund, U. (2012). Global physical activity levels: surveillance progress, pitfalls, and prospects. The Lancet, 380(9838), 247-57. doi:10.1016/ S0140-6736(12)60646-1

Juul, F., Martinez-Steele, E., Parekh, N., Monteiro, C. A., \& Chang, V. W. (2018). Ultra-processed food consumption and excess weight among US adults. British Journal of Nutrition, 120(01), 90-100. doi:10.1017/ S0007114518001046
Kumar, S., \& Kelly, A. S. (2017). Review of Childhood Obesity. Mayo Clinic Proceedings, 92(2), 251-65. doi:10.1016/j.mayocp.2016.09.017

Mariscal-Arcas, M., Rivas, A., Velasco, J., Ortega, M., Caballero, A. M., \& Olea-Serrano, F. (2009). Evaluation of the Mediterranean Diet Quality Index (KIDMED) in children and adolescents in Southern Spain. Public Health Nutrition, 12(09), 1408. doi:10.1017/S1368980008004126

Márquez-Sandoval, F., Bulló, M., Vizmanos, B., Casas-Agustench, P., \& Salas-Salvadó, J. (2008). Un patrón de alimentación saludable: la dieta mediterránea tradicional. Antropo, 16, 11-22. Recuperado de: https://genius.diba.cat/documents/10934/3667829/document1.pdf

Martins, A. P. B., Levy, R. B., Claro, R. M., Moubarac, J. C., \& Monteiro C. A. (2013). Participação crescente de produtos ultraprocessados na dieta brasileira (1987-2009). Revista de Saúde Pública, 47(4), 656-65. doi:10.1590/S0034-8910.2013047004968

Moher, D., Liberati, A., Tetzlaff, J., Altman, D. G., \& The PRISMA Group. (2009). Preferred Reporting Items for Systematic Reviews and MetaAnalyses: The PRISMA Statement. PLoS Medicine, 6(7), e1000097. doi:10.1371/journal.pmed.1000097

Mozaffarian, D., Appel, L. J., \& Van Horn, L. (2011). Components of a Cardioprotective Diet: New Insights. Circulation, 123(24), 2870-91. doi:10.1161/CIRCULATIONAHA.110.968735

Muros, J. J., Cofre-Bolados, C., Arriscado, D., Zurita, F., \& Knox, E. (2017). Mediterranean diet adherence is associated with lifestyle, physical fitness, and mental wellness among 10-y-olds in Chile. $\mathrm{Nu}$ trition, 35, 87-92. doi:10.1016/j.nut.2016.11.002

Navarro-Solera, M., González-Carrascosa, R., \& Soriano, J. M. (2014) Estudio del estado nutricional de estudiantes de educación primaria y secundaria de la provincia de Valencia y su relación con la adherencia a la Dieta Mediterránea. Revista Española de Nutrición Humana y Dietética, 18(2), 81. doi:10.14306/renhyd.18.2.65

Ng, M., Fleming, T., Robinson, M., Thomson, B., Graetz, N., Margono, C., ..., \& Gakidou, E. (2014). Global, regional, and national prevalence of overweight and obesity in children and adults during 1980-2013: a systematic analysis for the Global Burden of Disease Study 2013. The Lancet, 384(9945), 766-81. doi:10.1016/S0140-6736(14)60460-8

Owen, N., Sparling, P. B., Healy, G. N., Dunstan, D. W., \& Matthews, C. E. (2010). Sedentary Behavior: Emerging Evidence for a New Health Risk. Mayo Clinic Proceedings, 85(12), 1138-41. doi:10.4065/mcp.2010.0444

Pinel, C., Chacón, R., Castro, M., Espejo, T., Zurita, F., \& Cortés, A. (2017). Diferencias de género en relación con el Índice de Masa Corporal, calidad de la dieta y actividades sedentarias en niños de 10 a 12 años. Retos, 31, 176-80. Recuperado de: https://recyt.fecyt.es/index. $\mathrm{php} / \mathrm{retos} /$ article/view/49393/32170

Popkin, B. M. (2006a). Technology, transport, globalization and the nutrition transition food policy. Food Policy, 31(6), 554-69. doi:10.1016/ j.foodpol.2006.02.008

Popkin, B. M. (2006b). Global nutrition dynamics: the world is shifting rapidly toward a diet linked with noncommunicable diseases. The American Journal of Clinical Nutrition, 84(2), 289-98. doi:10.1093/ ajcn/84.1.289

Ratner, R., Durán, S., Garrido, M. J., Balmaceda, S., Jadue, L., \& Atalah E. (2013). Impacto de una intervención en alimentación y actividad física sobre la prevalencia de obesidad en escolares. Nutrición Hospitalaria, 28(5), 1508-14. doi:10.3305/nh.2013.28.5.6644

Roccaldo, R., Censi, L., D’Addezio, L., Toti, E., Martone, D., D’Addesa, D., ..., \& Cernigliaro, A. (2014). Adherence to the Mediterranean diet in Italian school children (The ZOOM8 Study). International Journal of Food Sciences and Nutrition, 65(5), 621-8. doi:10.3109/09637486. 2013.873887

Rosa, A., Carrillo, P. J., García, E., Pérez, J. J., Tárraga, L., \& Tárraga, P. J. (2018). Dieta mediterránea, estado de peso y actividad física en escolares de la Región de Murcia. Clínica e Investigación en Arteriosclerosis, 31(1), 1-7. doi:10.1016/j.arteri.2018.09.002

Rosa, A, García-Cantó, E., Rodríguez, P. L., Pérez, J. J., Tárraga, M. L., \& Tárraga, P. J. (2017). Actividad física, condición física y calidad de la dieta en escolares de 8 a 12 años. Nutrición Hospitalaria, 34(6), 129298. doi:10.20960/nh.813 
Rubio-López, N., Llopis-González, A., Picó, Y., \& Morales-Suárez-Varela, M. (2017). Dietary Calcium Intake and Adherence to the Mediterranean Diet in Spanish Children: The ANIVA Study. International Journal of Environmental Research and Public Health, 14(6), 637. doi:10.3390/ijerph14060637

Serra-Majem, L., Ribas, L., Ngo, J., Ortega, R. M., García, A., Pérez-Rodrigo, C., \& Aranceta, J. (2004). Food, youth and the Mediterranean diet in Spain. Development of KIDMED, Mediterranean Diet Quality Index in children and adolescents. Public Health Nutrition, 7(7), 931-5. doi:10.1079/PHN2004556

Sofi, F., Macchi, C., Abbate, R., Gensini, G. F., \& Casini, A. (2014). Mediterranean diet and health status: an updated meta-analysis and a proposal for a literature-based adherence score. Public Health Nutrition, 17(12), 2769-82. doi:10.1017/S1368980013003169

Trichopoulou, A., Kouris-Blazos, A., Wahlqvist, M. L., Gnardellis, C., Lagiou, P., Polychronopoulos, E., ..., \& Trichopoulos, D. (1995). Diet and overall survival in elderly people. BMJ, 311(7018), 1457-60. doi:10.1136/bmj.311.7018.1457

Vardavas, C. I., Linardakis, M. K., Hatzis, C. M., Saris, W. H. M., \& Kafatos, A. G. (2010). Cardiovascular disease risk factors and dietary habits of farmers from Crete 45 years after the first description of the Mediterranean diet. European Journal of Cardiovascular Prevention \& Rehabilitation, 17(4), 440-6. doi:10.1097/HJR.0b013e32833692ea Vassiloudis, I., Yiannakouris, N., Panagiotakos, D. B., Apostolopoulos, K., \& Costarelli, V. (2014). Academic performance in relation to adherence to the Mediterranean diet and energy balance behaviors in Greek primary schoolchildren. Journal of Nutrition Education and Behavior, 46(3), 164-70. doi.org:10.1016/j.jneb.2013.11.001

Vio, F., Salinas, J., Montenegro, E., González, C. C., \& Lera, L. (2014). Efecto de una intervención educativa en alimentación saludable en profesores y niños preescolares y escolares de la región de Valparaíso. Nutrición Hospitalaria, 29(6), 1298-304. doi:10.3305/nh.2014.29.6.7409 